\title{
Fluid-Structure Interaction using Nonconforming Finite Element Methods
}

\author{
Edward Swim and Padmanabhan Seshaiyer \\ Texas Tech University, Mathematics and Statistics (eswim@math.ttu.edu)
}

\begin{abstract}
Summary. Direct numerical solution of the highly nonlinear equations governing even the most simplified models of fluid-structure interaction requires that both the flow field and the domain shape be determined as part of the solution since neither is known a priori. To accomplish this, previous algorithms have decoupled the solid and fluid mechanics, solving for each separately and converging iteratively to a solution which satisfies both. In this paper, we describe a nonconforming finite element method which solves the problem of interaction between a viscous incompressible fluid and a structure whose deformation defines the interface between the two simultaneously. A general methodology is described for the model 2D problem and the algorithm is validated computationally for a one-dimensional example.
\end{abstract}

\section{Introduction}

Many applications from engineering and biological sciences, such as blood flow through arteries, require detailed simulation of an interaction between a fluid and an elastic membrane surrounding it. However, meshes generated for the purpose of analyzing the two materials may be incompatible and the cost of producing matching grids may be prohibitive. Much work has been done to build efficient numerical schemes using nonconforming finite element methods (Seshaiyer [2003], Seshaiyer and Suri [2000b], Seshaiyer and Suri [2000a], Belgacem et al. [2000], and references therein.) Thus far, these methods have focused on model problems where the governing equations on each sub-domain are the same, e.g., interaction between fluids (Chilton and Seshaiyer [2002]) or interaction between structures (Belgacem et al. [2003].)

Furthermore, it has been shown (Wan et al. [2003]) that even onedimensional models can be useful in predicting important characteristics of blood flow despite their simplicity. Our purpose here is to present a nonconforming finite element method for fluid-structure interaction problems which allows for both mesh refinement and degree enhancement independently on each component. The methodology is described for a two-dimensional model 
problem and the algorithm is computationally validated for a one-dimensional model.

\section{Governing equations}

Let $\mathbf{x}=\left(x_{1}, x_{2}\right)$. We consider a rectangular domain $\Omega$ which is divided into two sub-domains, $\Omega_{F}(t)$ and $\Omega_{S}(t)$, at any time $t$, as illustrated in Figure 1. We assume that a viscous incompressible fluid occupies $\Omega_{F}$ while an elastic solid material occupies $\Omega_{S}$. Initially, assume that $\Omega_{F}^{0} \equiv \Omega_{F}(0)=I \times I$, where $I=[0,1]$, and that $\Omega_{S}^{0} \equiv \Omega_{S}(0)=[1,2] \times I$. Let $\gamma\left(t, x_{2}\right)$ represent the interface between the two sub-domains.

We model the velocity $\mathbf{u} \in \mathbb{R}^{2}$ and pressure $p$ of the fluid using the NavierStokes equations,

$$
\sum_{i=1}^{2} \frac{\partial}{\partial x_{i}}\left[\mu_{F}\left(\frac{\partial u_{j}}{\partial x_{i}}+\frac{\partial u_{i}}{\partial x_{j}}\right)\right]-\frac{\partial p}{\partial x_{j}}=\rho_{F}\left(\frac{\partial u_{j}}{\partial t}+\nabla \cdot\left(u_{j} \mathbf{u}\right)-f_{j}\right)
$$

$j=1,2, \forall \mathbf{x} \in \Omega_{F}(t), t>0$. Here, $\mu_{F}$ is the dynamic viscosity, $\rho_{F}$ is the fluid density, and $\mathbf{f}=\left(f_{1}, f_{2}\right)$ is the applied force. Moreover, due to the incompressible nature of the fluid, the velocity must satisfy $\nabla \cdot \mathbf{u}=0$. Additionally, we model the displacement $\mathbf{d}$ of the solid from its initial position at time $t=0$ using the Navier-space equations,

$$
\sum_{i=1}^{2} \frac{\partial}{\partial x_{i}}\left[\mu_{S}\left(\frac{\partial d_{j}}{\partial x_{i}}+\frac{\partial d_{i}}{\partial x_{j}}\right)\right]+\lambda \frac{\partial}{\partial x_{j}}(\nabla \cdot \mathbf{d})=\rho_{S}\left(\frac{\partial^{2} d_{j}}{\partial t^{2}}-g_{j}\right),
$$

$j=1,2, \forall \mathbf{x} \in \Omega_{S}^{0}$. Here, $\lambda$ and $\mu_{S}$ are the Lamè coefficients, $\rho_{S}$ is the solid density, and $\mathbf{g}=\left(g_{1}, g_{2}\right)$ is the applied load on the structure. We impose homogeneous Dirichlet boundary conditions on $\partial \Omega_{F} \backslash \gamma$ and $\partial \Omega_{S} \backslash \gamma$ except on $\Gamma_{D W}$, the downwind boundary of $\Omega_{F}$. On this boundary, we only assume that the velocity $\mathbf{u}$ is known. In particular, we let $\left.\mathbf{u}\right|_{\Gamma_{D W}}=(0, \tilde{u})$.

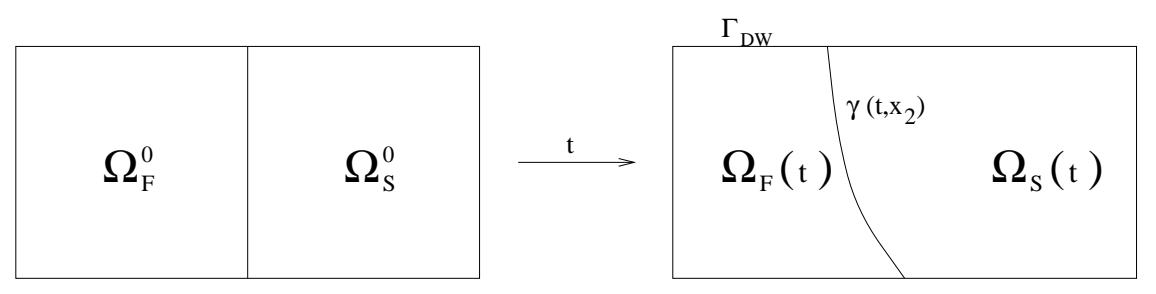

Fig. 1. Deformation of the fluid and solid sub-domains over time

Letting $\nu=\frac{\mu_{F}}{\rho_{F}}$, the kinematic viscosity of the fluid, and using incompressibility of the fluid, (1) reduces to 


$$
\frac{\partial u_{j}}{\partial t}-\nu \Delta u_{j}+\mathbf{u} \cdot \nabla u_{j}+\frac{\partial p}{\partial x_{j}}=f_{j}
$$

$j=1,2, \forall \mathbf{x} \in \Omega_{F}(t), t>0$. Similarly, if we let $\mu=\mu_{S}$ and $\varepsilon=\frac{\mu+\lambda}{\rho_{S}}$ then (2) becomes

$$
\frac{\partial^{2} d_{j}}{\partial t^{2}}-\mu \Delta d_{j}-\varepsilon \frac{\partial}{\partial x_{j}}(\nabla \cdot \mathbf{d})=g_{j}
$$

$j=1,2, \forall \mathbf{x} \in \Omega_{S}^{0}$.

The weak formulation for $(3)$ is to find $u_{j}$ satisfying

$$
\begin{aligned}
\int_{\Omega_{F}(t)}\left(\partial_{t} u_{j}\right) w_{F}^{(j)} d A+\nu \int_{\Omega_{F}(t)} \nabla u_{j} \cdot \nabla w_{F}^{(j)} d A-\nu \int_{\partial \Omega_{F}(t)} \frac{\partial u_{j}}{\partial \mathbf{n}} w_{F}^{(j)} d s \\
\quad+\int_{\Omega_{F}(t)}\left(\mathbf{u} \cdot \nabla u_{j}\right) w_{F}^{(j)} d A+\int_{\Omega_{F}(t)}\left(\partial_{x_{j}} p\right) w_{F}^{(j)} d A=\int_{\Omega_{F}(t)} f_{j} w_{F}^{(j)} d A
\end{aligned}
$$

for appropriate $w_{F}^{(j)} \in H^{1}\left(\Omega_{F}(t)\right), j=1,2$. Thus,

$$
a_{F}\left(\mathbf{u}, \mathbf{w}_{\mathbf{F}}\right)+b_{F}\left(p, \mathbf{w}_{\mathbf{F}}\right)+\tilde{c}\left(\mathbf{u}, \mathbf{w}_{\mathbf{F}}\right)+B_{F}\left(\boldsymbol{\Lambda}_{F}, \mathbf{w}_{\mathbf{F}}\right)=F_{F}\left(\mathbf{w}_{\mathbf{F}}\right),
$$

where

$$
\begin{gathered}
a_{F}\left(\mathbf{u}, \mathbf{w}_{\mathbf{F}}\right)=\nu \int_{\Omega_{F}} \sum_{j=1}^{2}\left(\nabla u_{j} \cdot \nabla w_{F}^{(j)}\right) d A, \\
b_{F}\left(p, \mathbf{w}_{\mathbf{F}}\right)=-\int_{\Omega_{F}} p\left(\nabla \cdot \mathbf{w}_{\mathbf{F}}\right) d A, \\
\tilde{c}\left(\mathbf{u}, \mathbf{w}_{\mathbf{F}}\right)=\int_{\Omega_{F}} \sum_{j=1}^{2}\left(\partial_{t} u_{j}+\mathbf{u} \cdot \nabla u_{j}\right) w_{F}^{(j)} d A, \\
B_{F}\left(\boldsymbol{\Lambda}_{F}, \mathbf{w}_{\mathbf{F}}\right)=-\int_{\gamma}\left(\boldsymbol{\Lambda}_{F} \cdot \mathbf{w}_{\mathbf{F}}\right) d s, \\
\boldsymbol{\Lambda}_{F}^{j}=\nu\left(\nabla u_{j} \cdot \mathbf{n}\right)-p n_{j},
\end{gathered}
$$

and

$$
F_{F}\left(\mathbf{w}_{\mathbf{F}}\right)=\int_{\Omega_{F}} \mathbf{f} \cdot \mathbf{w}_{\mathbf{F}} d A+\int_{\Gamma_{D W}} \tilde{u} w_{F}^{(2)} d s .
$$

Next, note that

$$
b_{F}(v, \mathbf{u})=0 \quad \forall v \in H^{1}\left(\Omega_{F}(t)\right) .
$$

And finally, $\forall w_{S}^{(j)} \in H^{1}\left(\Omega_{S}^{0}\right)$,

$$
\begin{array}{r}
\int_{\Omega_{S}^{0}}\left(\partial_{t t} d_{j}\right) w_{S}^{(j)} d A+\mu \int_{\Omega_{S}^{0}} \nabla d_{j} \cdot \nabla w_{S}^{(j)} d A-\mu \int_{\partial \Omega_{S}^{0}} \frac{\partial d_{j}}{\partial \mathbf{n}} w_{S}^{(j)} d s \\
-\varepsilon \int_{\Omega_{S}^{0}}\left[\partial_{x_{j}}(\nabla \cdot \mathbf{d})\right] w_{S}^{(j)} d A=\int_{\Omega_{S}^{0}} g_{j} w_{S}^{(j)} d A
\end{array}
$$


$j=1,2$. Thus,

$$
a_{S}\left(\mathbf{d}, \mathbf{w}_{\mathbf{s}}\right)+c_{S}\left(\mathbf{d}, \mathbf{w}_{\mathbf{s}}\right)+B_{S}\left(\boldsymbol{\Lambda}_{S}, \mathbf{w}_{\mathbf{s}}\right)=F_{S}\left(\mathbf{w}_{\mathbf{s}}\right),
$$

where

$$
\begin{gathered}
a_{S}\left(\mathbf{d}, \mathbf{w}_{\mathbf{S}}\right)=\int_{\Omega_{S}} \mu \sum_{j=1}^{2}\left(\nabla d_{j} \cdot \nabla w_{S}^{(j)}\right)+\varepsilon(\nabla \cdot \mathbf{d})\left(\nabla \cdot \mathbf{w}_{\mathbf{S}}\right) d A, \\
c_{S}\left(\mathbf{d}, \mathbf{w}_{\mathbf{S}}\right)=\int_{\Omega_{S}}\left(\partial_{t t} \mathbf{d}\right) \cdot \mathbf{w}_{\mathbf{S}} d A, \\
B_{S}\left(\boldsymbol{\Lambda}_{S}, \mathbf{w}_{\mathbf{S}}\right)=-\int_{\gamma} \boldsymbol{\Lambda}_{S} \cdot \mathbf{w}_{\mathbf{S}} d s, \\
\boldsymbol{\Lambda}_{S}{ }^{j}=\mu\left(\nabla d_{j} \cdot \mathbf{n}\right)+\varepsilon(\nabla \cdot \mathbf{d}) n_{j},
\end{gathered}
$$

and

$$
F_{S}\left(\mathbf{w}_{\mathbf{s}}\right)=\int_{\Omega_{S}} \mathbf{g} \cdot \mathbf{w}_{\mathbf{s}} d A .
$$

Now, on the interface

$$
\gamma\left(t, x_{2}\right)=1+d_{1}\left(t, 1, x_{2}\right),
$$

where $\mathbf{d}=\left(d_{1}, d_{2}\right)$, we wish to enforce continuity of the velocities,

$$
\mathbf{u}\left(t, \gamma\left(t, x_{2}\right), x_{2}\right)=\frac{\partial \mathbf{d}}{\partial t}\left(t, 1, x_{2}\right),
$$

and continuity of the flux,

$$
\left.\left(\boldsymbol{\Lambda}_{F}\right)\right|_{\gamma\left(t, x_{2}\right)}+\left.\left(\boldsymbol{\Lambda}_{S}\right)\right|_{\gamma\left(0, x_{2}\right)}=\mathbf{0} .
$$

To construct a two-field method, we set $\boldsymbol{\Lambda}_{N}=\left.\left(\boldsymbol{\Lambda}_{F}\right)\right|_{\gamma\left(t, x_{2}\right)}=-\left.\left(\boldsymbol{\Lambda}_{S}\right)\right|_{\gamma\left(0, x_{2}\right)}$. Then the global weak formulation is to find

$$
\left(\mathbf{u}, p, \mathbf{d}, \boldsymbol{\Lambda}_{N}\right) \in X_{N} \equiv\left[H^{1}\left(\Omega_{F}\right)\right]^{2} \times H^{1}\left(\Omega_{F}\right) \times\left[H^{1}\left(\Omega_{S}\right)\right]^{2} \times\left[H^{-\frac{1}{2}}(\gamma)\right]^{2}
$$

such that $\forall\left(\mathbf{w}_{\mathbf{F}}, v, \mathbf{w}_{\mathbf{S}}, \mathbf{\Psi}\right) \in X_{N}$,

$$
\begin{gathered}
a_{F}\left(\mathbf{u}, \mathbf{w}_{\mathbf{F}}\right)+b_{F}\left(p, \mathbf{w}_{\mathbf{F}}\right)+\tilde{c}\left(\mathbf{u}, \mathbf{w}_{\mathbf{F}}\right)+B_{F}\left(\boldsymbol{\Lambda}_{N}, \mathbf{w}_{\mathbf{F}}\right)=F_{F}\left(\mathbf{w}_{\mathbf{F}}\right), \\
a_{S}\left(\mathbf{d}, \mathbf{w}_{\mathbf{S}}\right)+c_{S}\left(\mathbf{d}, \mathbf{w}_{\mathbf{S}}\right)-B_{S}\left(\boldsymbol{\Lambda}_{N}, \mathbf{w}_{\mathbf{S}}\right)=F_{S}\left(\mathbf{w}_{\mathbf{S}}\right), \\
b_{F}(v, \mathbf{u})=0,
\end{gathered}
$$

and

$$
G\left(\mathbf{u}-\frac{\partial}{\partial t} \mathbf{d}, \Psi\right)=0
$$

where

$$
G(\mathbf{u}, \Psi)=\int_{\gamma} \mathbf{u} \cdot \boldsymbol{\Psi} d s .
$$

To solve a fully coupled fluid-structure interaction problem simultaneously for both $\mathbf{u}$ and $\mathbf{d}$, one must compute the solution to (8)-(11) and then extrapolate a piecewise linear approximation for the interface $\gamma$ at each time step. 


\section{A one-dimensional model problem}

Imposing (possibly nonconforming) meshes on $\Omega_{F}^{0}$ and $\Omega_{S}^{0}$ and assuming a piecewise linear interface $\gamma\left(t, x_{2}\right)$ corresponding to the mesh for $\Omega_{F}(t)$ as in Figure 2, the spatially discrete problem is closely related to a collection of one-dimensional problems of the following form.

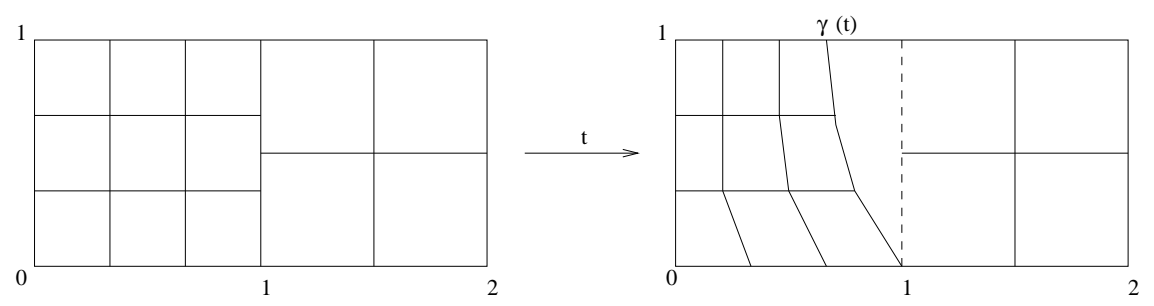

Fig. 2. Evolution of a nonconforming mesh for $\Omega$

We consider a coupled system (Grandmont et al. [2001]) for a velocity $u$ satisfying a modified Burgers' equation and displacement $d$ satisfying a wave equation, e.g.,

$$
\frac{\partial u}{\partial t}-\nu \frac{\partial^{2} u}{\partial x^{2}}+\frac{3}{2} u \frac{\partial u}{\partial x}=f, \quad x \in(0, \gamma(t)),
$$

where $u(t, 0)=0$, and

$$
\frac{\partial^{2} d}{\partial t^{2}}-\mu \frac{\partial^{2} d}{\partial x^{2}}=g, \quad x \in(1,2),
$$

where $d(t, 2)=0$ and $d(0, x)=0, x \in(1,2)$, with interface $\gamma(t)=1+d(t, 1)$, as illustrated in Figure 3. Again, we enforce continuity of velocities, i.e.,

$$
u(t, \gamma(t))=\frac{\partial d}{\partial t}(t, 1)
$$

and continuity of flux,

$$
\nu \frac{\partial u}{\partial x}(t, \gamma(t))=\mu \frac{\partial d}{\partial x}(t, 1) .
$$

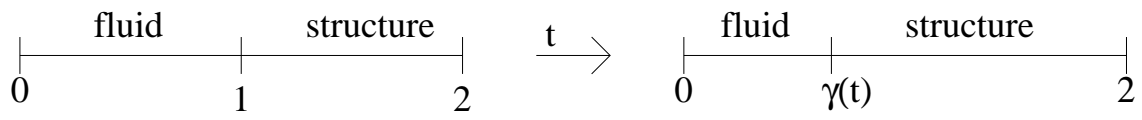

Fig. 3. Evolution of a one-dimensional domain 
Employing an arbitrary Lagrangian-Eulerian (ALE) formulation for the fluid equation and an implicit formulation for the interface position, the discrete variational problem is to find $\bar{u}^{n+1}, d^{n+1}$, and $\Gamma^{n+1}$ satisfying

$$
\begin{gathered}
\left(\bar{u}^{n+1}, \phi\right)_{n}-\Delta t \nu \phi\left(\Gamma^{n}\right) \partial_{x} \bar{u}^{n+1}\left(\Gamma^{n}\right)+\Delta t \nu\left(\partial_{x} \bar{u}^{n+1}, \partial_{x} \phi\right)_{n} \\
+\Delta t\left(\left(u^{n}-w^{n}\right) \partial_{x} \bar{u}^{n+1}, \phi\right)_{n}+\frac{1}{2} \Delta t\left(\bar{u}^{n+1} \partial_{x} u^{n}, \phi\right)_{n} \\
=\left(u^{n}, \phi\right)_{n}+\Delta t\left(f^{n+1}, \phi\right)_{n} \\
\frac{1}{\Delta t}\left(d^{n+1}, \psi\right)+\Delta t \mu \psi(1) \partial_{x} d^{n+1}(1)+\Delta t \mu\left(\partial_{x} d^{n+1}, \partial_{x} \psi\right) \\
=\frac{1}{\Delta t}\left(2 d^{n}-d^{n-1}, \psi\right)+\Delta t\left(g^{n+1}, \psi\right) \\
\bar{u}^{n+1}\left(\Gamma^{n}\right)=\frac{1}{\Delta t}\left(d^{n+1}-d^{n}\right)(1) \\
\nu \partial_{x} \bar{u}^{n+1}\left(\Gamma^{n}\right)=\mu \partial_{x} d^{n+1}(1)
\end{gathered}
$$

and

$$
\Gamma^{n+1}=1+D^{n+1}(1)
$$

where $\bar{u}^{n+1}(x)=u^{n+1}\left(x+w^{n}(x) \Delta t\right)$ and $w^{n}(x)=\frac{x}{\Gamma^{n}} u^{n}\left(\Gamma^{n}\right)$ for $n=1,2, \ldots$, $\Gamma^{n}$ is the time-discrete approximation of $\gamma\left(t^{n}\right),(\cdot, \cdot)_{n}$ is the scalar product on $L^{2}\left(0, \Gamma^{n}\right)$, and $(\cdot, \cdot)$ is the scalar product on $L^{2}(1,2)$.

\section{Computational experiments}

Using hierarchic basis functions (Szabo and Babuska [1991]), we construct finite element approximations $\bar{U}^{n} \approx \bar{u}^{n}$ and $D^{n} \approx d^{n}$ and solve the resulting linear system for $\bar{U}^{n+1}, D^{n+1}$, and $\Gamma^{n+1}$.

For our experiments, we let $\mu=2$ and consider an exact solution for (12)-(15) given by

$$
\begin{gathered}
u(t, x)=\frac{-2 \pi x^{2} \cos (\pi t)}{(2-\sin (\pi t))^{2}}, \\
d(t, x)=\frac{1}{2} x(x-2) \sin (\pi x t),
\end{gathered}
$$

and

$$
\gamma(t)=1-\frac{1}{2} \sin (\pi t)
$$

where $\nu(t)=\frac{1}{2} \mu t\left(1-\frac{1}{2} \sin (\pi t)\right)$. Note that both $u$ and $d$ are nonlinear.

Assuming a uniform grid and linear basis functions, let $M$ be the number of subintervals allowed on the interval $\left(0, \Gamma^{n}\right)$ and let $N$ be the number of subintervals on $(1,2)$. Figure 4 compares the relative error of our method whenever $M=N$ to the case $M=N-1$ as the degrees of freedom increase. 
The relative error under the $L^{2}$ norm is plotted against the total degrees of freedom. Panel (a) shows the results for the fluid velocity and panel (b) shows the results for the structural displacement. Furthermore, it was previously shown (Grandmont et al. [2001]) that the consistency error for both $u(t, x)$ and $d(t, x)$ is of order $\Delta t$. Table 1 illustrates this property for our test problem.
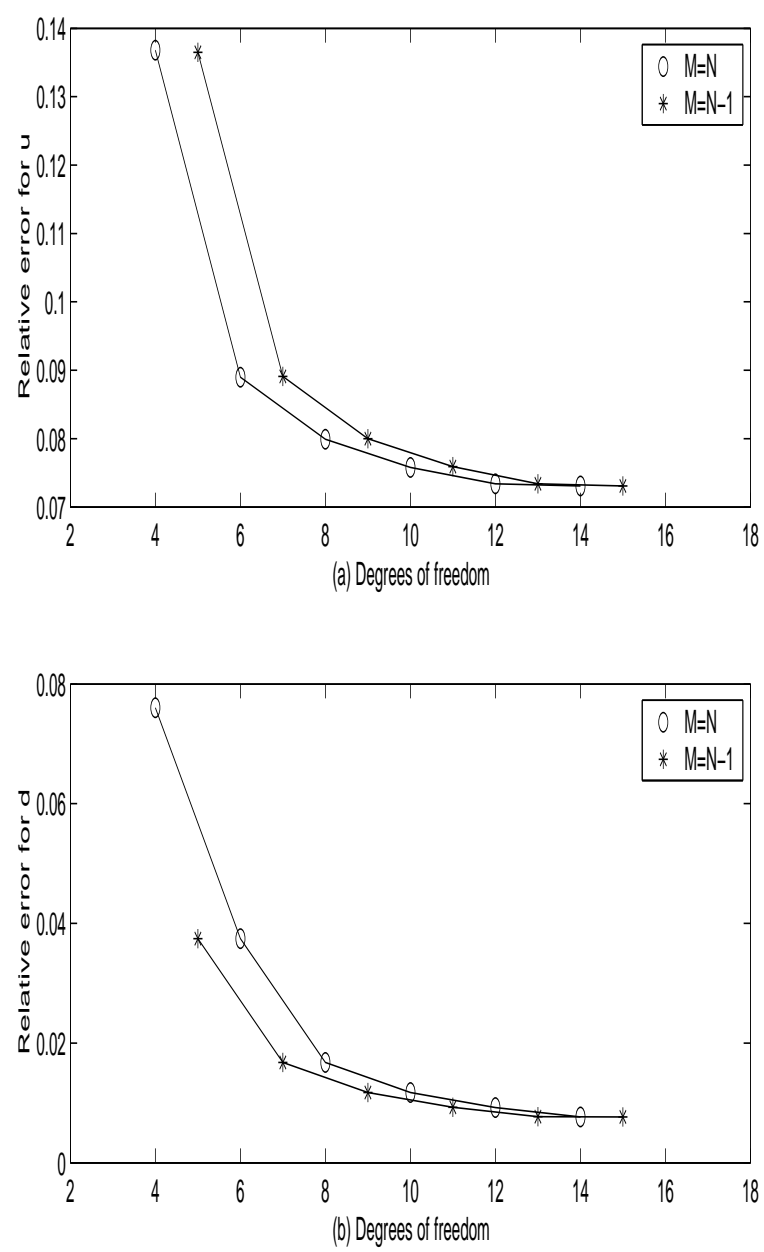

Fig. 4. Convergence of relative error on a uniform mesh

In conclusion, our numerical results provide confidence that this method will be successful when extended to higher-dimensional problems. To do this, one only needs to employ the ALE formulation along a given line segment $\left\{\left(x_{1}, x_{2}\right): 0 \leq x_{1} \leq \gamma\left(t, x_{2}\right)\right\}$. We intend to present an implementation of this method for $2 \mathrm{D}$ problems in the $h p$ context in future work. 
Table 1. Relative $L^{2}$ error for fluid velocity and structure displacement as $\Delta t$ decreases

\begin{tabular}{ccc}
\hline$\Delta t$ & $\|u-U\|_{L^{2}\left(0, \Gamma^{n}\right)} /\|u\|_{L^{2}\left(0, \Gamma^{n}\right)}\|d-D\|_{L^{2}(1,2)} /\|d\|_{L^{2}(1,2)}$ \\
\hline 0.1 & 0.445914 & 0.169221 \\
0.05 & 0.243088 & 0.070792 \\
0.025 & 0.170837 & 0.031750 \\
0.00625 & 0.133612 & 0.009507 \\
\hline
\end{tabular}

Acknowledgement. This work was sponsored in part by the ARCS Foundation of Lubbock and by the National Science Foundation under grant DMS 0207327.

\section{References}

F. B. Belgacem, L. Chilton, and P. Seshaiyer. The $h p$ mortar finite element method for the mixed elasticity and stokes problems. Comp. Math. App., 46:35-55, 2003.

F. B. Belgacem, P. Seshaiyer, and M. Suri. Optimal convergence rates of $h p$ mortar finite element methods for second-order elliptic problems. RAIRO Math. Mod. Numer. Anal., 34:591-608, 2000.

L. Chilton and P. Seshaiyer. The $h p$ mortar domain decomposition method for problems in fluid mechanics. Int. Jour. Numer. Meth. Fluids, 40:1561-1570, 2002.

C. Grandmont, V. Guimet, and Y. Maday. Numerical analysis of some decoupling techniques for the approximation of the unsteady fluid structure interaction. Math. Mod. Meth. App. Sci., 11:1349-1377, 2001.

P. Seshaiyer. Stability and convergence of nonconforming $h p$ finite-element methods. Comp. Math. App., 46:165-182, 2003.

P. Seshaiyer and M. Suri. $h p$ submeshing via non-conforming finite element methods. Comp. Meth. Appl. Mech. Engrg., 189:1011-1030, 2000a.

P. Seshaiyer and M. Suri. Uniform $h p$ convergence results for the mortar finite element method. Math. Comp., 69:521-546, 2000b.

B. Szabo and I. Babuska. Finite element analysis. Wiley. New York, 1991.

J. Wan, B. Steele, S. Spicer, S. Strohband, G. Feijoo, T. Hughes, and C. Taylor. A one-dimensional finite element method for simulation-based medical planning for cardiovascular disease. Comp. Meth. Biomech. Biomed. Engrg., 2003. Submitted. 